\title{
A SUBCLASS OF UNIVALENT FUNCTIONS
}

\author{
R. M. GOEL and BEANT SINGH MEHROK
}

(Received 23 December 1980, revised 19 June 1981)

Communicated by R. Vyborny

\begin{abstract}
Sharp results for the coefficient estimates, distortion theorems, radius of convexity, arc-length and area of the image curve are obtained for the class $R(A, B)$ of regular functions whose derivative is subordinate to $(1+A z) /(1+B z),-1 \leqslant B<A \leqslant 1$, in the unit disc $E=\{z:|z|<1\}$. We also establish a convolution theorem for this class.

1980 Mathematics subject classification (Amer. Math. Soc.): 30 A 32, 30 A 34, 30 A 42.

Keywords and phrases: quasi-subordination, subordination, majorization, subclass of univalent functions, convolution (Hadamard product).
\end{abstract}

\section{Introduction}

Let $U$ denote the class of functions

$$
w(z)=\sum_{k=1}^{\infty} c_{k} z^{k}
$$

which are regular in $E=\{z:|z|<1\}$ and satisfying there the conditions $w(0)=0$ and $|w(z)|<1$.

Let $S$ denote the class of functions

$$
f(z)=z+\sum_{k=2}^{\infty} a_{k} z^{k}
$$

regular and univalent in $E$.

Copyright Australian Mathematical Society 1983 
Let $R(A, B)$ denote the class of functions $f(z)=z+\sum_{k=2}^{\infty} a_{k} z^{k}$ which are regular in $E$ and satisfying there

$$
f^{\prime}(z) \prec \frac{1+A z}{1+B z}, \quad-1 \leqslant B<A \leqslant 1, z \in E .
$$

Obviously $R(+1,-1)$ coincides with $R$, the class of functions $f(z)=z+$ $\sum_{k=2}^{\infty} a_{k} z^{k}$ regular in $E$ and satisfying $\operatorname{Re} f^{\prime}(z)>0, z \in E$. Thus $R(A, B)$ is a subclass of $R(1,-1)$. To avoid repetition we lay down, once for all, that $-1 \leqslant B<$ $A \leqslant 1$, and $z \in E$.

Let us set

$$
f^{\prime}(z)=P(z)=1+\sum_{k=1}^{\infty} p_{k} z^{k} .
$$

Then by definition of subordination, $f \in R(A, B)$ if and only if $f^{\prime}(z)$ has the representation

$$
f^{\prime}(z)=P(z)=\frac{1+A w(z)}{1+B w(z)}, \quad w \in U .
$$

An easy computation shows that $f \in R(A, B)$ if and only if

$$
\left|f^{\prime}(z)-1\right|<\left|A-B f^{\prime}(z)\right| \text {. }
$$

Alexander [1] and Wolff [17] made an early study of the class $R$. It follows from the Noshiro-Warschawski theorems $[12,16]$ that functions of the class $R$ are equivalent in $E$. Hence $R(A, B)$ is a subclass of $S$.

MacGregor [9] investigated the properties of the class $R$, and subsequently, the same author [10] studied the subclass $R(1)$ of $R$ of regular functions $f(z)$ satisfying the condition

$$
\left|f^{\prime}(z)-1\right|<1 \text {. }
$$

The first author [5,6] developed some properties of the subclass $S(\alpha)$ of $R$ of regular functions $f(z)$ which satisfy the condition

$$
\left|f^{\prime}(z)-\alpha\right|<\alpha, \quad\left(\alpha>\frac{1}{2}\right) .
$$

Padmanabhan [13] investigated the subclass $R(\alpha)$ of $R$ of regular functions $f(z)$ satisfying

$$
\left|\frac{f^{\prime}(z)-1}{f^{\prime}(z)+1}\right|<\alpha, \quad 0<\alpha \leqslant 1 .
$$

Capling and Causey [4] also studied the class $R(\alpha)$ and improved some of the results due to Padmanabhan [13].

The following observations are obvious:

(i) $R(1,-1) \equiv R$,

(ii) $R(1,0) \equiv R(1)$, 
(iii) $R(1,1 / \alpha-1) \equiv S(\alpha),\left(\alpha>\frac{1}{2}\right)$,

(iv) $R(\alpha,-\alpha) \equiv R(\alpha),(0<\alpha \leqslant 1)$.

Thus, $R(A, B)$ contains all the above mentioned classes, and therefore the view of Brickman [3, page 341], "idea of subordination has unified the geometric theory of functions" is strengthened.

In this paper, we obtain sharp result for coefficient estimates, distortion theorems, radius of convexity, arc-length and area of the image curve for the class $R(A, B)$. We also prove that if

$$
f(z)=z+\sum_{n=2}^{\infty} a_{n} z^{n}, \quad h(z)=z+\sum_{n=2}^{\infty} b_{n} z^{n}
$$

belong to $R(A, B)$, then so does $F(z)=z+\frac{1}{2} \sum_{n=2}^{\infty} n a_{n} b_{n} z^{n}$.

Results due to MacGregor [9, 10], Padmanabhan [13], Capling and Causey [4] and the first author [5, 6] follow as special cases from our theorems.

\section{Some preliminary lemmas}

LEMMA 1. If $g(z)$ and $G(z)$ are regular in $|z|<1$ and $g(z)$ is subordinate to $G(z)$ $(g(z) \prec G(z))$ with $g(0)=G(0)$, then for $\lambda>0,0<r<1$,

$$
\int_{0}^{2 \pi}\left|g\left(r e^{i \theta}\right)\right|^{\lambda} d \theta \leqslant \int_{0}^{2 \pi}\left|G\left(r e^{i \theta}\right)\right|^{\lambda} d \theta
$$

This lemma is due to Littlewood and its proof can be found in [7, page 484, Theorem 2; 8(1944), Theorem 210].

Robertson [14] introduced the concept of quasi-subordination. Let $g(z)$ and $G(z)$ be analytic in $E$. Let $\phi(z)$ be analytic and $|\phi(z)| \leqslant 1$ in $E$, such that $g(z) / \phi(z)$ is regular and subordinate to $G(z)$, for $z \in E$. Then $g(z)$ is said to be quasi-subordinate to $G(z)$, written as $g(z) \prec_{q} G(z), z \in E$.

An equivalent condition for this is

$$
g(z)=\phi(z) G(w(z)), \quad|\phi(z)| \leqslant 1, w \in U, z \in E .
$$

If $\phi(z)=1$, then $g(z)=G(w(z))$ so that $g(z)<G(z)$ in $E$. If $w(z)=z$, then $g(z)=\phi(z) G(z)$, we say that $g(z)$ is majorized by $G(z)$ and we write it as $g(z) \ll G(z), z \in E$.

LEMMA 2. If $g(z)=\sum_{k=0}^{\infty} d_{k} z^{k} \prec_{q} G(z)=\sum_{k=0}^{\infty} D_{k} z^{k}$, then

$$
\sum_{k=0}^{n}\left|d_{k}\right|^{2} \leqslant \sum_{k=0}^{n}\left|D_{k}\right|^{2} \quad(n=0,1,2, \ldots) \text {. }
$$

This lemma is due to Robertson [14]. 
In particular (2.2) holds also when

(i) $g(z) \prec G(z)$,

(ii) $g(z) \ll G(z)$.

By the application of Lemma 2, we establish

Lemma 3. For $f \in R(A, B)$, if $f^{\prime}(z)=P(z)=1+\sum_{k=1}^{\infty} p_{k} z^{k}$, then

$$
\left|p_{n}\right| \leqslant(A-B), \quad n \geqslant 1 \text {. }
$$

The bounds are sharp.

Proof. From (1.5) we have

$$
\sum_{k=1}^{\infty} p_{k} z^{k}=w(z)\left[(A-B)-B \sum_{k=1}^{\infty} p_{k} z^{k}\right] .
$$

By the application of Lemma 2(2.2), we get

$$
\sum_{k=1}^{n}\left|p_{k}\right|^{2} \leqslant(A-B)^{2}+B^{2} \sum_{k=1}^{n-1}\left|p_{k}\right|^{2}
$$

or

$$
\left|p_{n}\right|^{2} \leqslant(A-B)^{2}-\left(1-B^{2}\right) \sum_{k=1}^{n-1}\left|p_{k}\right|^{2} \leqslant(A-B)^{2} .
$$

This yields (2.3).

Equality signs in (2.3) are attained for the functions $P_{n}(z)$ defined by

$$
P_{n}(z)=\frac{1+A \delta z^{n}}{1+B \delta z^{n}}, \quad|\delta|=1
$$

In order to determine the radius of convexity, we need the following two lemmas.

LeMma 4. For $w \in U$ and $|z|=r$, we have

$$
\left|z w^{\prime}(z)-w(z)\right| \leqslant \frac{r^{2}-|w(z)|^{2}}{1-r^{2}} .
$$

This result is due to Singh and Goel proved in [15].

LEMMA 5. Let

$$
p(z)=\frac{1+B w(z)}{1+A w(z)}, \quad w \in U
$$


Then for $|z|=r<1$,

(2.5) $\operatorname{Re}\left(A p(z)+\frac{B}{p(z)}\right)+\frac{r^{2}|A p(z)-B|^{2}-|1-p(z)|^{2}}{\left(1-r^{2}\right)|p(z)|}$

$\leqslant \begin{cases}\frac{A B(A+B) r^{2}-4 A B r+(A+B)}{(1-A r)(1-B r)}, & R_{1} \leqslant R_{0}, \\ \frac{2}{\left(1-r^{2}\right)}\left[\left(1-A B r^{2}\right)-\left((1-A)(1-B)\left(1+A r^{2}\right)\left(1+B r^{2}\right)\right)^{1 / 2}\right], & R_{1} \geqslant R_{0}, A \neq 1,\end{cases}$

where

$$
R_{1}=\frac{1-B r}{1-A r}, \quad R_{0}^{2}=\frac{(1-B)\left(1+B r^{2}\right)}{(1-A)\left(1+A r^{2}\right)}
$$

The bounds are sharp.

Proof. It is easy to see that the transformation

$$
p(z)=\frac{1+B w(z)}{1+A w(z)}
$$

maps $|w(z)| \leqslant r$ onto the circle $|p(z)-a| \leqslant d$, where

$$
a=\frac{\left(1-A B r^{2}\right)}{\left(1-A^{2} r^{2}\right)} \quad \text { and } \quad d=\frac{(A-B) r}{\left(1-A^{2} r^{2}\right)} \text {. }
$$

Putting $p(z)=R e^{i \theta}\left(-\frac{\pi}{2}<\theta<\frac{\pi}{2}\right)$ and denoting the left hand side of (2.5) by $T(R, \theta)$, we get

$$
\begin{aligned}
T(R, \theta)= & (A R+B / R) \cos \theta+\frac{2\left(1-A B r^{2}\right) \cos \theta}{\left(1-r^{2}\right)} \\
& -\frac{\left(1-A^{2} r^{2}\right) R}{\left(1-r^{2}\right)}-\frac{\left(1-B^{2} r^{2}\right)}{\left(1-r^{2}\right) R}
\end{aligned}
$$

For extreme values of $T(R, \theta), \partial T / \partial R=0=\partial T / \partial \theta$ which yield respectively

$$
\cos \theta=\frac{\left(1-A^{2} r^{2}\right)-\left(1-B^{2} r^{2}\right) / R^{2}}{\left(1-r^{2}\right)\left(A-B / R^{2}\right)}, \quad\left(-\frac{\pi}{2}<\theta<\frac{\pi}{2}\right),
$$

and

$$
L(R) \sin \theta=0,
$$


where

$$
L(R)=(A R+B / R)+\frac{2\left(1-A B r^{2}\right)}{\left(1-r^{2}\right)} .
$$

Now we prove that $L(R)$ remains positive. If $B \geqslant 0, A>0$, then $L(R)>0$. We now consider the case when $B<0$. The following cases arise.

Case I. $B<0, A \geqslant 0$. Using the fact that $0<\cos \theta \leqslant 1$, it follows from (2.7) that

$$
\frac{1-B^{2} r^{2}}{1-A^{2} r^{2}}<R^{2} \leqslant \frac{(1-B)\left(1+B r^{2}\right)}{(1-A)\left(1+A r^{2}\right)}
$$

$L^{\prime}(R)=\left(A-B / R^{2}\right)>0$ and hence $L(R)$ attains its minimum value at

$$
R=\left(\frac{1-B^{2} r^{2}}{1-A^{2} r^{2}}\right)^{1 / 2}=R_{2}, \quad \text { say. }
$$

Now

$$
\begin{aligned}
L\left(R_{2}\right)= & {\left[\left(1-r^{2}\right)(A+B)+2\left(1-A^{2} r^{2}\right)^{1 / 2}\left(1-B^{2} r^{2}\right)^{1 / 2}\right] } \\
& \times \frac{\left(1-A B r^{2}\right)}{\left(1-r^{2}\right)\left[\left(1-A^{2} r^{2}\right)\left(1-B^{2} r^{2}\right)\right]^{1 / 2}}
\end{aligned}
$$

which is positive provided

$$
\left(1-r^{2}\right)(A+B)+2\left(\left(1-A^{2} r^{2}\right)\left(1-B^{2} r^{2}\right)\right)^{1 / 2}>0 .
$$

If $(A+B) \geqslant 0$, there is nothing to prove, so we assume $(A+B)<0$. (2.12) will hold if

$$
4\left(1-A^{2} r^{2}\right)\left(1-B^{2} r^{2}\right)-(A+B)^{2}\left(1-r^{2}\right)^{2}>0
$$

or if

$$
\left[(1+B)\left(1+A r^{2}\right)(1+A)\left(1+B r^{2}\right)\right]\left[2\left(1+A B r^{2}\right)-(A+B)\left(1+r^{2}\right)\right]>0
$$

which is always true.

Case II. $B<0, A<0$. Consider the case when $L^{\prime}(R)=\left(A-B / R^{2}\right)<0$. Since $0<\cos \theta \leqslant 1$, it follows from (2.7) that

$$
\frac{(1-B)\left(1+B r^{2}\right)}{(1-A)\left(1+A r^{2}\right)} \leqslant R^{2}<\frac{1-B^{2} r^{2}}{1-A^{2} r^{2}} .
$$

An easy computation would show that this does not hold. 
Now, consider the case when $\left(A-B / R^{2}\right) \geqslant 0$. If $A-B / R^{2}=0$, then from (2.7), we have

$$
R^{2}=\frac{\left(1-B^{2} r^{2}\right)}{\left(1-A^{2} r^{2}\right)}=\frac{B}{A}
$$

and it implies $(A-B)\left(1+A B r^{2}\right)=0$ which is evidently not possible. Thus the only case needed to be considered is when $\left(A-B / R^{2}\right)>0$. Therefore, by $(2.10)$,

$$
\frac{1-B^{2} r^{2}}{1-A^{2} r^{2}}<R^{2}<B / A .
$$

The minimum value of $L(R)$ occurs at $R=R_{2}$ and $L\left(R_{2}\right)>0$ if

$$
\left(1-r^{2}\right)(A+B)+2\left[\left(1-A^{2} r^{2}\right)\left(1-B^{2} r^{2}\right)\right]^{1 / 2}>0
$$

which holds as proved in Case I (when $A+B$ is negative). For extreme values, from (2.8) and (2.7), we get

$$
\theta=0, \pi ; \quad R^{2}=\frac{(1-B)\left(1+B r^{2}\right)}{(1-A)\left(1+A r^{2}\right)}=R_{0}^{2}, \text { say. }
$$

It can be easily verified that $T(R, \theta)$ attains its maximum value at $(\theta=0$, $\left.R=R_{0}\right)$. So

$$
\begin{aligned}
T(R, \theta) & \leqslant T\left(R_{0}, 0\right) \\
& =\frac{2}{\left(1-r^{2}\right)}\left[\left(1-A B r^{2}\right)-\left((1-A)(1-B)\left(1+A r^{2}\right)\left(1+B r^{2}\right)\right)^{1 / 2}\right] .
\end{aligned}
$$

It is easy to see that $R_{0} \geqslant a-d=(1+B r) /(1+A r)$. But $R_{0}$ is not always less than or equal to $a+d$. In case $R_{0} \notin[a=d, a+d]$, the maximum of $T(R, 0)$ is attained at

$$
R=R_{1}=(a+d)=\frac{1-B r}{1-A r}
$$

and equals

$$
T\left(R_{1}, 0\right)=\frac{A B(A+B) r^{2}-4 A B r+(A+B)}{(1-A r)(1-B r)}, \quad R_{1} \leqslant R_{0} .
$$

If $R_{1} \leqslant R_{0}$, equality sign in (2.5) holds for the function

$$
p(z)=\frac{1+B z}{1+A z} .
$$

If $R_{1} \geqslant R_{0}, A \neq 1$, equality sign in (2.5) holds for the function

$$
p_{0}(z)=\frac{1-(1+B) z \cos \theta+B z^{2}}{1-(1+A) z \cos \theta+A z^{2}}
$$


where

$$
R_{0}=\frac{1-(1+B) r \cos \theta+B r^{2}}{1-(1+A) r \cos \theta+A r^{2}}
$$

Hence the lemma is established.

\section{Coefficient estimates}

THEOREM 3.1. Let $f \in R(A, B)$ then

$$
\left|a_{n}\right| \leqslant \frac{(A-B)}{n}, \quad n \geqslant 2 .
$$

The bounds are sharp for the functions $f_{(n-1)}(z)$ defined by

$$
f_{(n-1)}(z)=\int_{0}^{z}\left(\frac{1+A \delta t^{n-1}}{1+B \delta t^{n-1}}\right) d t, \quad|\delta|=1 .
$$

Proof. (3.1) follows on equating the coefficients of $z^{n}$ in (1.4) and then using (2.3).

THEOREM 3.2. If $f \in R(A, B)$ and if $\mu$ is a complex number, then

$$
\left|a_{3}-\mu a_{2}^{2}\right| \leqslant \frac{(A-B)}{3} \max \left\{1,\left|B+\frac{3(A-B)}{4} \mu\right|\right\} .
$$

The estimate is sharp.

Proof. On equating the coefficients of $z^{2}$ and $z^{3}$ in (1.5), we get

$$
\begin{aligned}
& c_{1}=\frac{2 a_{2}}{(A-B)}, . \\
& c_{2}=\frac{3}{(A-B)}\left[a_{3}+\frac{4 B}{3(A-B)} a_{2}^{2}\right] .
\end{aligned}
$$

Also

$$
\left|c_{2}\right| \leqslant 1-\left|c_{1}\right|^{2} \text {. }
$$

Therefore, for every complex number $v$, we have

$$
\begin{aligned}
\left|c_{2}-v c_{1}^{2}\right| & \leqslant\left|c_{2}\right|+|v|\left|c_{1}\right|^{2} \\
& \leqslant 1+(|v|-1)\left|c_{1}\right|^{2} \\
& \leqslant \max \{1,|v|\},
\end{aligned}
$$


since $\left|c_{1}\right| \leqslant 1$. The estimate (3.6) is sharp for $w(z)=z$ and $w(z)=z^{2}$ respectively for $|v| \leqslant 1$ and $|v|<1$. From (3.4) and (3.5) we have

$$
\left|a_{3}-\mu a_{2}^{2}\right|=\frac{(A-B)}{3}\left|c_{2}-v c_{1}^{2}\right|
$$

where

$$
\mu=\frac{4}{3(A-B)}(v-B)
$$

or

$$
v=B+\frac{3(A-B) \mu}{4} .
$$

(3.7) in conjunction with (3.6) and (3.7), yields (3.3). (3.3) is sharp, being attained for the function $f_{1}(z)$ and $f_{2}(z)$ defined, respectively, by

$$
f_{1}^{\prime}(z)=\frac{1+A z}{1+B z} \text { and } f_{2}^{\prime}(z)=\frac{1+A z^{2}}{1+B z^{2}} .
$$

\section{Distortion theorems}

THEOREM 4.1. Let $f \in R(A, B)$, then for $|z|=r<1$,

$$
\begin{aligned}
& \left|f^{\prime}(z)\right| \leqslant \frac{1+A r}{1+B r} ; \\
& \operatorname{Re} f^{\prime}(z) \geqslant \frac{1-A r}{1-B r} ; \\
& |f(z)| \leqslant \begin{cases}\frac{A}{B}\left[r+\left(\frac{1}{A}-\frac{1}{B}\right) \log (1+B r)\right], & B \neq 0, \\
r+\frac{A}{2} r^{2}, & B=0,\end{cases} \\
& |f(z)| \geqslant \begin{cases}\frac{A}{B}\left[r-\left(\frac{1}{A}-\frac{1}{B}\right) \log (1-B r)\right], & B \neq 0, \\
r-\frac{A}{2} r^{2}, & B=0 .\end{cases}
\end{aligned}
$$

All the estimates are sharp.

Proof. From (1.5), it is easy to establish (4.1) and (4.2). Using (4.1),

$$
|f(z)| \leqslant \int_{0}^{r}\left|f^{\prime}\left(t e^{i \beta}\right)\right| d t \leqslant \int_{0}^{r} \frac{1+A t}{1+B t} d t
$$


which yields (4.3). Again using (4.2),

$$
|f(z)| \geqslant \int_{0}^{r} \operatorname{Re} f^{\prime}\left(t e^{i \beta}\right) d t \geqslant \int_{0}^{r} \frac{1-A t}{1-B t} d t
$$

which gives (4.4). (4.1) and (4.3) are sharp, being attained for the function

$$
f_{1}(z)= \begin{cases}\frac{A}{B}\left[z+\left(\frac{1}{A}-\frac{1}{B}\right) \log (1+B z)\right], & B \neq 0, \\ z+\frac{A}{2} z^{2}, & B=0 .\end{cases}
$$

(4.2) and (4.4) are sharp for the function

$$
f_{(1)}(z)= \begin{cases}\frac{A}{B}\left[z-\left(\frac{1}{A}-\frac{1}{B}\right) \log (1-B z)\right], & B \neq 0, \\ z-\frac{A}{2} z^{2}, & B=0 .\end{cases}
$$

Let $W$ be any complex number such that

$$
|W|< \begin{cases}\frac{A}{B}\left[r-\left(\frac{1}{A}-\frac{1}{B}\right) \log (1-B r)\right], & B \neq 0 \\ r-\frac{A}{2} r^{2}, & B=0 .\end{cases}
$$

By Rouche's Theorem it follows that $f(z)$ and $f(z)-W$ have the same number of zeros in $|z|<r$, that is, precisely one. Hence we have the following:

CoRollaRY. Every function $f(z)$ in $R(A, B)$ maps $E$ onto a domain which covers the disc

$$
|W|< \begin{cases}\frac{A}{B}\left[1-\left(\frac{1}{A}-\frac{1}{B}\right) \log (1-B)\right], & B \neq 0, \\ 1-\frac{A}{2}, & B=0 .\end{cases}
$$

\section{Argument of $f^{\prime}(z)$}

THEOREM 5.1. If $f \in R(A, B)$ then

$$
\left|\arg f^{\prime}(z)\right| \leqslant \sin ^{-1} \frac{(A-B) r}{1-A B r^{2}}, \quad|z|=r .
$$

The result is sharp. 
Proof. It is easy to show that $f^{\prime}(z)=(1+A w(z)) /(1+B w(z))$ maps $|w(z)|$ $\leqslant r$ onto the circle

$$
\left|f^{\prime}(z)-\frac{\left(1-A B r^{2}\right)}{1-B^{2} r^{2}}\right| \leqslant \frac{(A-B) r}{\left(1-B^{2} r^{2}\right)}
$$

(5.1) is an immediate consequence of (5.2). The result is sharp, being attained for the function $f_{0}(z)$ defined by

$$
f_{0}^{\prime}(z)=\frac{1+A \delta z}{1+B \delta z}
$$

where

$$
\delta=\frac{z}{r}\left[\frac{-(A+B) r+i\left(\left(1-A^{2} r^{2}\right)\left(1-B^{2} r^{2}\right)\right)^{1 / 2}}{1+A B r^{2}}\right]
$$

\section{Convex set of functions}

THEOREM 6.1. If $f$ and $h \in R(A, B)$, then

$$
\lambda f+(1-\lambda) h \in R(A, B), \quad(0 \leqslant \lambda \leqslant 1) .
$$

Proof. By definition,

$$
\begin{aligned}
f^{\prime}(z) & <\frac{1+A z}{1+B z}, \\
h^{\prime}(z) & <\frac{1+A z}{1+B z} .
\end{aligned}
$$

Since $(1+A z) /(1+B z)$ is convex univalent in $E$, it follows by a result due to Bernardi [2, page 57, Example 2] that

$$
\lambda f^{\prime}(z)+(1-\lambda) h^{\prime}(z) \prec \frac{1+A z}{1+B z} .
$$

Hence

$$
\lambda f^{\prime}+(1-\lambda) h^{\prime} \in R(A, B) .
$$

\section{Radius of convexity}

THEOREM 7.1. Let $f \in R(A, B)$, then

(i) for $A_{0} \leqslant A \leqslant 1, f(z)$ is convex in $|z|<r_{0}$, where $r_{0}$ is the smallest positive root of

$$
A B r^{2}-2 A r+1=0
$$


(ii) for $-1<A \leqslant A_{0}, f(z)$ is convex in $|z|<r_{1}$, where $r_{1}$ is the smallest positive root of

(7.2)

$$
\begin{gathered}
A\left(1-r^{2}\right)-\left[\left(1-A B r^{2}\right)-\left((1-A)(1-B)\left(1+A r^{2}\right)\left(1+B r^{2}\right)\right)^{1 / 2}\right]=0 ; \\
A_{0}=\frac{\left(2+B-2 B^{2}\right)+\left(20-36 B+21 B^{2}-4 B^{3}\right)^{1 / 2}}{2\left(4-2 B-B^{2}\right)} .
\end{gathered}
$$

The results are sharp.

Proof. Differentiating logarithmically, (1.5) yields

$$
1+\frac{z f^{\prime \prime}(z)}{f^{\prime}(z)}=1+(A-B) \frac{z w^{\prime}(z)}{(1+A w(z))(1+B w(z))} .
$$

(7.3) together with Lemma 4 gives

$$
\begin{aligned}
\operatorname{Re}\left(1+\frac{z f^{\prime \prime}(z)}{f^{\prime}(z)}\right) \geqslant 1+(A-B) \\
\quad \times\left[\operatorname{Re} \frac{w(z)}{(1+A w(z))(1+B w(z))}-\frac{r^{2}-|w(z)|^{2}}{\left(1-r^{2}\right)|(1+A w(z))(1+B w(z))|}\right] .
\end{aligned}
$$

Putting $p(z)=(1+B w(z)) /(1+A w(z))$, and using Lemma 5,

$$
\operatorname{Re}\left(1+\frac{z f^{\prime \prime}(z)}{f^{\prime}(z)}\right)
$$

$$
\geqslant\left\{\begin{array}{l}
\frac{2 A}{A-B}-\frac{A B(A+B) r^{2}-4 A B r+(A+B)}{(A-B)(1-A r)(1-B r)}, \quad R_{1} \leqslant R_{0}, \\
\frac{2 A}{A-B}-2\left[\frac{\left(1-A B r^{2}\right)-\left((1-A)(1-B)\left(1+A r^{2}\right)\left(1+B r^{2}\right)\right)^{1 / 2}}{\left(1-r^{2}\right)(A-B)}\right], \\
\quad R_{1} \geqslant R_{0}, A \neq 1 .
\end{array}\right.
$$

(7.1) and (7.2) follow by equating the right hand sides of (7.5) to zero.

The equation $R_{0}=R_{1}$ yields

$$
A B r^{4}-2 A B r^{3}+[2(A+B)-A B-1] r^{2}-2 r+1=0 .
$$

Elimination of $r$ between (7.1) and (7.6) leads to

$$
\left(4-2 B-B^{2}\right) A^{2}-\left(2+B-2 B^{2}\right) A-(1-B)^{2}=0 .
$$


(7.7), on verification of the signs, yields

$$
A=\frac{\left(2+B-2 B^{2}\right)+\left(20-36 B+21 B^{2}-4 B^{3}\right)^{1 / 2}}{2\left(4-2 B-B^{2}\right)}=A_{0}, \text { say. }
$$

The results are sharp, being attained respectively, for the functions $f_{1}(z)$ and $f_{\theta}(z)$ defined by

$$
f_{1}^{\prime}(z)=\frac{1+A z}{1+B z}, \quad f_{\theta}^{\prime}(z)=\frac{1-(1+A) z \cos \theta+A z^{2}}{1-(1+B) z \cos \theta+B z^{2}},
$$

where $\theta$ is defined by (2.13).

REMARK 1. Radii of convexity for the classes $R, R(1)$ and $S(\alpha)$, at once, follow from (7.1).

REMARK 2. On taking $A=\alpha, B=-\alpha(0<\alpha \leqslant 1)$ in (7.7), we get $\alpha^{4}-4 \alpha^{3}-$ $4 \alpha^{2}+4 \alpha+1=0$ which gives

$$
\alpha=\frac{\left(2^{1 / 2}-1\right)\left(3^{1 / 2}+1\right)}{2^{1 / 2}}=\alpha_{0}, \quad \text { say. }
$$

Hence

(i) for $\alpha_{0} \leqslant \alpha \leqslant 1, f(z)$ maps $|z|<\left(2^{1 / 2}-1\right) / \alpha$ onto a convex domain;

(ii) for $0<\alpha \leqslant \alpha_{0}, f(z)$ maps

$$
|z|<\left[\frac{\left(\alpha^{2}-1\right)+\left(\left(1-\alpha^{2}\right)\left(1+4 \alpha-\alpha^{2}\right)\right)^{1 / 2}}{2 \alpha(1+\alpha)}\right]^{1 / 2}
$$

onto a convex domain. This result was established by Padmanabhan in [13] and also by Capling and Causey in [4].

\section{Arc-length and area of the image curve}

THEOREM 8.1. Let $f \in R(A, B)$ and $L_{r}(f)$ denotes the length of the image of $|z|=r$ under $f(z), 0<r<1$, then

$$
L_{r}(f) \leqslant \begin{cases}\pi r\left[\left|\frac{A+B}{B}\right|-\frac{(A-B)}{|B|}-2 \frac{(A-B)}{\pi B} \log \left(\frac{1-B r}{1+B r}\right)\right], & B \neq 0, \\ r \int_{0}^{2 \pi}\left|1+A r e^{i \theta}\right| d \theta, & B=0 .\end{cases}
$$

The results are sharp. 
Proof. In Lemma 1 , set $g(z)=f^{\prime}(z), G(z)=(1+A z) /(1+B z)$ and $\lambda=1$. Then

$$
\int_{0}^{2 \pi}\left|f^{\prime}\left(r e^{i \theta}\right)\right| d \theta \leqslant \int_{0}^{2 \pi}\left|\frac{1+A r e^{i \theta}}{1+B r e^{i \theta}}\right| d \theta
$$

Now

$$
\begin{aligned}
L_{r}(f) & =\int_{|z|=r}\left|f^{\prime}(z)\right||d z| \\
& =\int_{0}^{2 \pi}\left|f^{\prime}\left(r e^{i \theta}\right)\right| r d \theta
\end{aligned}
$$

By (8.2),

$$
\begin{aligned}
& L_{r}(f) \leqslant r \int_{0}^{2 \pi}\left|\frac{1+A r e^{i \theta}}{1+B r e^{i \theta}}\right| d \theta \\
& =r \int_{0}^{2 \pi}\left|\begin{array}{c}
\frac{A+B}{2 B}-\frac{(A-B)\left(1-B^{2} r^{2}\right)}{2 B\left(1+2 B r \cos \theta+B^{2} r^{2}\right)} \\
+i \frac{(A-B) r \sin \theta}{1+2 B r \cos \theta+B^{2} r^{2}}
\end{array}\right| d \theta \\
& \leqslant \pi r\left|\frac{A+B}{B}\right|+\frac{(A-B) r}{2|B|} \int_{0}^{2 \pi} \frac{\left(1-B^{2} r^{2}\right)}{1+2 B r \cos \theta+B^{2} r^{2}} d \theta \\
& +(A-B) r \int_{0}^{2 \pi} \frac{r|\sin \theta|}{1+2 B r \cos \theta+B^{2} r^{2}} d \theta \\
& =\pi r\left|\frac{A+B}{B}\right|+\frac{\pi(A-B) r}{|B|}-\frac{(A-B) r}{B} \int_{0}^{\pi} \frac{-2 B r \sin \theta}{1+2 B r \cos \theta+B^{2} r^{2}} d \theta \\
& =\pi r\left[\left|\frac{A+B}{B}\right|+\frac{(A-B)}{|B|}-2 \frac{(A-B)}{\pi B} \log \left(\frac{1-B r}{1+B r}\right)\right] \text {. }
\end{aligned}
$$

For $B=0$, result is trivial.

The extremal function $f_{0}(z)$ is given by

$$
f_{0}^{\prime}(z)=\frac{1+A \delta z}{1+B \delta z}, \quad|\delta|=1
$$

COROLlaRY. For the class $R(\alpha)$, we deduce, from (8.1),

$$
L_{r}(f) \leqslant 2 \pi r+4 r \log \left(\frac{1+\alpha r}{1-\alpha r}\right) .
$$

This is a result established by Capling and Causey [4]. 
THEOREM 8.2. If $f \in R(A, B)$, and if $A_{r}(f)$ denotes the area of image of $|z|=r$ under $f(z), 0<r<1$, then

$$
A_{r}(f) \leqslant \begin{cases}\pi r^{2}\left[\left(1-\frac{(A-B)^{2}}{B^{2}}\right)-\frac{(A-B)^{2}}{B^{4} r^{2}} \log \left(1-B^{2} r^{2}\right)\right], & B \neq 0, \\ \pi r^{2}\left[1+\frac{A^{2} r^{2}}{2}\right], & B=0 .\end{cases}
$$

The inequalities are sharp.

(8.4) are direct consequences of Lemma $1(\lambda=2)$ and interior area theorem. Equality sign is attained for the function $f_{0}(z)$ defined by (8.3).

COROLlary. For the class $R(\alpha)$, we have from (8.4),

$$
A_{r}(f) \leqslant \pi r^{2}\left[-3-\frac{4}{\alpha^{2} r^{2}} \log \left(1-\alpha^{2} r^{2}\right)\right] .
$$

This is a result due to Capling and Causey [4].

\section{Convolution}

THEOREM 9.1. If $f(z)=z+\sum_{n=2}^{\infty} a_{n} z^{n}$ and $h(z)=z+\sum_{n=2}^{\infty} b_{n} z^{n}$ belong to the class $R(A, B)$, then so does

$$
F(z)=z+\frac{1}{2} \sum_{n=2}^{\infty} n a_{n} b_{n} z^{n}
$$

Proof. Since $f \in R(A, B)$, it follows by (1.6) that $\left|f^{\prime}(z)-1\right|<\left|A-B f^{\prime}(z)\right|$. It is equivalent to

$$
\left|f^{\prime}(z)-b\right| \leqslant C
$$

where $b=(1-A B) /\left(1-B^{2}\right), C=(A-B) /\left(1-B^{2}\right)$. It is easy to see that $1-b<C \leqslant b$. We know that if $H(z)=\sum_{n=0}^{\infty} h_{n} z^{n}$ is regular for $|z|<1$ and $|H(z)| \leqslant M$, then, by [11, page 101],

$$
\sum_{n=0}^{\infty}\left|h_{n}\right|^{2} \leqslant M^{2}
$$

Applying (9.2) to (9.1), we get $(1-b)^{2}+\sum_{n=2}^{\infty} n^{2}\left|a_{n}\right|^{2}<C^{2}$ or

$$
\sum_{n=2}^{\infty} n^{2}\left|a_{n}\right|^{2}<\frac{(A-B)^{2}}{\left(1-B^{2}\right)^{2}} \text {. }
$$


Similarly

$$
\sum_{n=2}^{\infty} n^{2}\left|b_{n}\right|^{2}<\frac{(A-B)^{2}}{\left(1-B^{2}\right)^{2}}
$$

Now

$$
\begin{aligned}
\left|F^{\prime}(z)-b\right|^{2}= & \left|(1-b)+\frac{1}{2} \sum_{n=2}^{\infty} n^{2} a_{n} b_{n} z^{n-1}\right|^{2} \\
\leqslant & (1-b)^{2}+(1-b) \sum_{n=2}^{\infty} n^{2}\left|a_{n}\right|\left|b_{n}\right| r^{n-1}+\frac{1}{4}\left|\sum_{n=2}^{\infty} n^{2} a_{n} b_{n} z^{n-1}\right|^{2} \\
\leqslant & (1-b)^{2}+(1-b)\left(\sum_{n=2}^{\infty} n^{2}\left|a_{n}\right|^{2} r^{n-1}\right)^{1 / 2}\left(\sum_{n=2}^{\infty} n^{2}\left|b_{n}\right|^{2} r^{n-1}\right)^{1 / 2} \\
& +\frac{1}{4}\left(\sum_{n=2}^{\infty} n^{2}\left|a_{n}\right|^{2} r^{n-1}\right)\left(\sum_{n=2}^{\infty} n^{2}\left|b_{n}\right|^{2} r^{n-1}\right) \\
\leqslant & (1-b)^{2}+(1-b)\left(\sum_{n=2}^{\infty} n^{2}\left|a_{n}\right|^{2}\right)^{1 / 2}\left(\sum_{n=2}^{\infty} n^{2}\left|b_{n}\right|^{2}\right)^{1 / 2} \\
& +\frac{1}{4}\left(\sum_{n=2}^{\infty} n^{2}\left|a_{n}\right|^{2}\right)\left(\sum_{n=2}^{\infty} n^{2}\left|b_{n}\right|^{2}\right) \\
\leqslant & (1-b)^{2}+(1-b) \frac{(A-B)^{2}}{\left(1-B^{2}\right)^{3}}+\frac{1}{4} \frac{(A-B)^{4}}{\left(1-B^{2}\right)^{4}} \\
= & \frac{B^{2}(A-B)^{2}}{\left(1-B^{2}\right)^{2}}+\frac{B(A-B)^{3}}{\left(1-B^{2}\right)^{3}}+\frac{1}{4} \frac{(A-B)^{4}}{\left(1-B^{2}\right)^{4}}
\end{aligned}
$$

$F \in R(A, B)$ if

$$
\frac{B^{2}(A-B)^{2}}{\left(1-B^{2}\right)^{2}}+\frac{B(A-B)^{3}}{\left(1-B^{2}\right)^{3}}+\frac{1}{4} \frac{(A-B)^{4}}{\left(1-B^{2}\right)^{4}}<\frac{(A-B)^{2}}{\left(1-B^{2}\right)^{2}} .
$$

This gives on simplification, $(A+B)<2$ which is true. Hence $F \in R(A, B)$.

REMARK. The first author [6] proved this theorem for the class $S(\alpha)$.

\section{Acknowledgement}

The authors would like to thank the referee for a number of suggestions. 


\section{References}

[1] J. W. Alexander, 'Functions which map the interior of the unit circle upon simple regions', Ann. of Math. 17 (1915), 12-22.

[2] S. D. Bernardi, 'Special classes of subordinate functions', Duke Math. J. 33 (1966), 55-67.

[3] L. Brickman, 'Subordinate families of analytic functions', Illinois J. Math. 15 (1971), 241-248.

[4] T. R. Capling and W. M. Causey, 'A class of univalent functions', Proc. Amer. Math. Soc. 39 (1973), 357-361.

[5] R. M. Goel, 'A class of univalent functions whose derivatives have positive real part in the unit disc', Nieuw Arch. Wisk. (3), 15 (1967), 55-63.

[6] R. M. Goel, 'A class of analytic functions whose derivatives have positive real part in the unit disc', Indian J. Math. 3 (1971), 141-145.

[7] J. E. Littlewood, 'On inequalities in the theory of functions', Proc. London Math. Soc. (2), 23 (1925), 481-519.

[8] J. E. Littlewood, Lectures on the theory of functions, pp. 163-185 (Oxford Univ. Press, London, 1944).

[9] T. H. MacGregor, 'Functions whose derivative has a positive real part', Trans. Amer. Math. Soc. 104 (1962), 532-537.

[10] T. M. MacGregor, 'A class of univalent functions', Proc. Amer. Math. Soc. 15 (1964), 311-317.

[11] Z. Nehari, Conformal mapping (McGraw Hill, New York, 1952).

[12] K. Noshiro, 'On the theory of schlicht functions', J. Fac. Sci. Hokkaido Univ. (1), 2 (1934-35), 129-155.

[13] K. S. Padmanabhan, 'On a certain class of functions whose derivatives have a positive real part in the unit disc', Ann. Polon. Math. 23 (1970), 73-81.

[14] M. S. Robertson, 'Quasi-subordinate functions', Mathematical essays dedicated to $A$. $J$. MacIntyre, pp. 311-330 (Ohio Univ. Press, Athens, Ohio, 1967).

[15] V. Singh and R. M. Goel, 'On radii of convexity and starlikeness of some classes of functions', J. Math. Soc. Japan 23 (1971), 323-339.

[16] S. S. Warschawaki, 'On the higher derivatives at the boundary in conformal mappings', Trans. Amer. Math. Soc. 8 (1935), 310-340.

[17] J. Wolff, ' $L$ 'intégrale d'une fonction holomorphe et à partie réelle positive dans un demiplan est univalente', C. R. Acad. Sci. Paris Sér A-B 198 (1934), 1209-1210.

\section{Department of Mathematics}

Panjabi University

Patiala-147002

(Panjab State)

India 